\title{
Oropharyngeal Cancer pT4b TNM Finding v6
}

National Cancer Institute

\section{Source}

National Cancer Institute. Oropharyngeal Cancer pT 4b TNM Finding v6. NCI Thesaurus. Code C64444.

Oropharyngeal cancer with tumor invading the lateral pterygoid muscle, pterygoid plates, lateral nasopharynx, or skull base or encases carotid artery. (from AJCC 6th Ed.) 